\title{
The following abstracts were presented as posters at the 2017 NEI Congress
}

\section{DISCLAIMER}

The material presented in this section contains the abstracts submitted as part of the poster session at the 2016 NEI Congress, Colorado Springs, CO, November 9-12, 2017.

The abstracts have not been peer reviewed as part of the standard processes of CNS Spectrums and may contain promotional content that does not represent the view or policies of the journal or publisher. The abstracts are not approved for continuing education credit. Poster content and the views expressed therein are those of the presenting entity and not the Editors of CNS Spectrums, Cambridge University Press or of the Neuroscience Education Institute. Authors supply a standard disclosure form to the conference organizers which they retain. 


\section{The following abstracts were presented as posters at the 2017 NEI Congress}

Congratulations to the scientific poster winners:

$\mathbf{1}^{\text {st }}$ Place: \#178-Gender Differences in Prodromal Symptoms of Dementia

$2^{\text {nd }}$ Place: \#146-Effect of Heroin Use on Changes of Brain Functions As Measured by fMRI, a Systematic Review

$3^{\text {rd }}$ Place: \#185-Second Generation Antipsychotics and Catatonia: A Literature Review

100

\section{Reciprocal Relationship Between Olfactory Ability and Olfactory Hallucination}

Usama Bardan' (4th-year medical student); Stefany Kress, Bsc' (Medical Student); and Alan R. Hirsch, MD'

${ }^{1}$ Smell and Taste Treatment and Research Foundation, Chicago, IL

ABSTRACT: Introduction: Transient fluctuation of smell concurrent with phantosmia has not been reported. Four such cases are presented.

METHODS: Case 1: A 27-year-old left handed (pathological) female, 7 years prior to presentation, noted constant olfactory hallucinations of dried blood and rotten sour eggs, level 8/10 in intensity.

Results: Without phantosmia: Alcohol Sniff Test (AST): 14 (hyposmia). Brief Smell Identification Test (BSIT): 10 (normosmia). Retronasal Smell Index: 0 (abnormal).

With phantosmia: AST: 1 (anosmia). BSIT: 12 (normosmia). Retronasal Smell Index: 0 (abnormal). Normal 72-hour EEG and MRI.

Case 2: A 19 year old right-handed woman presented with a 4 month history of unpleasant, fruity, rotten phantosmia occurring three times a day, 6-7/10 in intensity.

Results: Without phantosmia: Pocket Smell Test (PST): 3 (normosmia). AST: 30 (normosmia). With phantosmia: AST: 13 (hyposmia). CT scan: normal. MRI: normal. Case 3: A 40 year old right-handed female presented with ashtray/cigarette phantosmia , occurring 10 times a day, lasting seconds to all day, 10/10 in intensity. Her sense of smell is normal except when the phantosmia is present, during which time it decreases to $70 \%$ of normal.

Results: Without phantosmia: BSIT: 11 (normosmia). AST: 30 (normosmia). With phantosmia: AST: 11 (hyposmia). PST: 3 (normosmia). EEG: normal. MRI: few subcortical white matter hyperintensities on flair imaging.

Case 4: A 60 year old right handed male with type 1 diabetes mellitus presented with four months of phantosmia of sweet tobacco, level $8 / 10$ in severity, involves both nostrils, lasting 10 seconds and occurring two times a day. Over time, the hallucinated odor changed to a soapy smell 2-3/10 in intensity.

Results: Without phantosmia: AST: 6 (hyposmia). BSIT: 8 (hyposmia). With phantosmia: AST: 1 (anosmia). PST: 2 (hyposmia). CT scan: normal.

DISCUSSION: Olfactory ability should be assessed in those with phantosmia, both during and in the absence of hallucinated odors, to detect transient olfactory deficits in order to direct treatment towards this condition.

\section{4}

\section{Valproate-Induced Hyperammonemic Encephalopathy: Case Studies}

Dan Matthews, $M D^{\prime}$; and Glenda Matthews, $M D^{2}$

${ }^{1}$ Corporate Director Of Neuropsychiatric Services, Universal Health Services, Inc, Austin, TX

${ }^{2}$ Private Practice, Austin, Texas

ABSTRACT: BACKGROUND: Hyperammonemia and carnitine deficiency with concomitant encephalopathy have been reported to result from valproic acid administration (Coulter DL, J Child Neurol 1991Jan; 6(1); 7-14 and Mock, CM, et al, Am J Health Syst Pharm, 2012 Jan; 69(1):35-9). Although there have been numerous publications regarding this adverse event in the neurology literature, there have been very few reports published in the psychiatric literature. The reported incidence of hyperammonemia in children treated with valproate is $19 \%$. It is important that prescribers be aware of the risk of valproic acid induced hyperammonemic encephalopathy, as well as its diagnosis and management.

OBJECTIVE: The current study explores the feasibility of reversing Valproate Induced Hyperammonemic Encephalopathy (VHE) by discontinuing valproic acid and normalizing the carnitine level via L-carnitine supplementation. 